\title{
METABOLIC EVALUATION OF PATIENTS WITH RECURRENT AND MULTIPLE RENAL STONES: A PROSPECTIVE STUDY
}

\author{
CHIDAMBARAM DHANDAPANI*, JYOTHI SUJATHA SHIBULAL*, KUPPURAJAN NARAYANASAMY
}

Department of Urology, Kovai Medical Centre and Hospital, Coimbatore, Tamil Nadu, India. Email: jyoash91@gmail.com

Received: 18 August 2016, Revised and Accepted: 09 September 2016

\section{ABSTRACT}

Objective: Renal stone disease has become an important clinical condition worldwide, and it results from a combination of several factors which are biochemical, epidemiologic, and genetic in origin. Preventive measures are very important concerning stone disease and these measures greatly depend on the type of stones. Performing a metabolic evaluation of stone patients to identify metabolic abnormality, if any, and treating the same helps reduce the recurrence of stones. To identify and correct the metabolic risk factors of patients presenting with either recurrent, bilateral, or multiple stones and thereby reduce the recurrence of stones.

Methods: A hospital-based prospective observational study, including collection of essential data of 100 patients and analyzing them for any metabolic abnormality.

Results: Out of the 100 patients, 73 were males and 27 were females. The frequency of stone formation was high in the age group 51-60, even though age is not a significant factor. Only 55 patients underwent metabolic evaluation, in which 23 patients (41.8\%) had a metabolic abnormality. The common abnormality found in this geographical area was hyperuricosuria (29.1\%) followed by hypercalciuria (7.3\%). Hypercalciuria was not statistically significant, but hyperuricosuria and acidic urine $\mathrm{pH}$ were significant factors that contribute to stone formation.

Conclusion: Metabolic evaluation is a must for renal stone patients which greatly help reduce the risk of stone recurrence. Hyperuricemia was observed to be the major abnormality followed by hypercalciuria. Acidic urine $\mathrm{pH}$ and low urine volume were other significant risk factors.

Keywords: Nephrolithiasis, Metabolic evaluation, Hyperuricosuria, Hypercalciuria.

(C) 2016 The Authors. Published by Innovare Academic Sciences Pvt Ltd. This is an open access article under the CC BY license (http://creativecommons. org/licenses/by/4. 0/) DOI: http://dx.doi.org/10.22159/ajpcr.2016.v9s3.14776

\section{INTRODUCTION}

Renal calculus disease or nephrolithiasis is an important clinical condition which may result in hematuria and severe abdominal pain. Stones, when bilateral, can obstruct urinary flow which sometimes can lead to end stage renal disease, but nephrolithiasis is not an essential risk factor for incident chronic kidney disease [1]. A kidney stone, also called a renal calculus or nephrolith, is a mass of crystals which when formed together, create a hard lump in one or both the kidneys. It is formed from minerals present in the urine and can be present for a long time without causing any problems or symptoms When they move, they cause discomfort and severe pain (renal colic) in the back or abdomen that radiates to the groin. Other associated symptoms include vomiting, fever, blood in urine, pus in urine, and painful urination. The majority of renal stones can pass out of the body in the urine without any help, whereas some require intervention to remove them [2].

Stone disease has become a relatively common disorder, and its prevalence is greatly increasing worldwide. The diagnosis of renal stones is usually based on the symptoms, urine testing, and also medical imaging. Blood tests may also be useful. There are different types of stones based on their composition: Calcium oxalate $(80 \%)$, calcium phosphate $(5-10 \%)$, uric acid (5-10\%), oxalate $(5 \%)$, cysteine $(1-2 \%)$, and struvite stones (10-15\%) [3]. High dietary intake of animal protein, sodium, refined sugars, fructose, and high cola drinks can greatly contribute to the increased risk of developing renal calculi. Hereditary factors are also related to stone formation. People with certain medical conditions, such as hypertension, diabetes, gout, kidney infection, and those who take certain medications or supplements are at risk for renal stones [4].

Metabolic evaluation of renal stones, especially bilateral, multiple, or recurrent stones have become an important part of this clinical condition as it helps to identify the major metabolic abnormalities contributing to the formation of stones. It has been established from various studies that a stone former would have one or more metabolic abnormalities contributing to stone formation, which when treated properly can greatly help reduce the recurrence of stones. Reliable stone analysis and basic metabolic evaluation are greatly recommended in all the patients after stone passage. Metabolic screening allows diet related stone identification, points out stone risk factors, and identifies patient's comorbidity. People who have significant metabolic abnormalities are more at risk for stone formation, whereas people who frequently get stones are observed to have more significant metabolic abnormality [5].

Metabolic evaluation usually includes tests for serum calcium, phosphorus, uric acid, creatinine, blood urea nitrogen (BUN), electrolytes, stone analysis, and urinalysis, including urine $\mathrm{pH}$. Apart from this, 24-hrs urine collections on a random diet with components of urine such as urine volume, calcium, sodium, creatinine, citrate, uric acid, and oxalate are also an essential part of metabolic evaluation [6].

The general preventive measures for renal calculi includes increasing the fluid intake to more than 3 litres/day, reducing the intake of animal proteins, dairy products, and purine-rich foods, maintaining a healthy weight, adequate fruits, vegetables and fiber intake, regular exercise, and making self-free from other medical conditions such as diabetes, hypertension, gout, and hyperparathyroidism [2].

Here, in this geographical area, renal stone patients are common and there are plenty of them consulting with complaints of severe abdominal pain and other symptoms. In this study, the major focus was given to patients who were diagnosed with bilateral, recurrent, and multiple stones, and single stone formers were excluded as this 
study was conducted to evaluate the metabolic abnormalities related to stone formation. Single stones occur as a result of either structural abnormality of the urinary tract or lifestyle pattern of the patients such as low water intake, high protein intake, occupation in hot places, and obesity. Single stone usually is not related to any metabolic abnormality. Any metabolic abnormality in an individual can result in staghorn calculi or multiple/bilateral stone. If this abnormality is left untreated, it can lead to recurrence of stones. The common metabolic abnormalities occurring in such patients are hypercalciuria, hyperuricosuria, hyperoxaluria, and hypocitraturia. An individual can have one or more than one metabolic abnormality. Thus, evaluation of the metabolic abnormality and treating the same can greatly reduce the incidence of stones and improve the quality of life of the patients.

\section{METHODS}

The study was conducted over a time of 7 months, starting from January to July 2016, in the Kovai Medical Center and Hospital, Coimbatore, after ethical approval has been obtained from the Ethics Committee of the hospital in the month of January 2016. This was a hospital-based prospective observational study, enrolling 100 patients presenting with bilateral, multiple, or recurrent stones, to the Department of Urology of the hospital. The main tool used to collect the patient details was a properly designed data collection form which included variables such as age, sex, body mass index (BMI), dietary pattern, family history, past history, average water intake, alcohol intake, type of stone, diagnostic tests, 24 hrs urine analysis components, serum calcium, serum uric acid, serum creatinine, random blood sugar (RBS), and BUN and also treatment methods used. Out of the 100 patients selected, 73 were males and 27 were females. 55 patients had bilateral stones and around 23 presented with recurrence.

The inclusion criteria were patients having bilateral/multiple stones and recurrent stone formers, whereas single stone, infection stones, and first-time stone formers (FTSFs) were excluded.

SPSS software was used to statistically analyze the data, and the main statistical method used to find out the significance of each variable as risk for stone formation was Chi-square test. A p $<0.05$ was considered significant. Descriptive statistics was also used to obtain the variable versus percent graphs.

A wide literature survey was also conducted to obtain literatures relating and supporting the present study.

\section{RESULTS}

A total of 100 patients who came with either bilateral, multiple, or recurrent renal stones to the Urology Department of $\mathrm{KMCH}$ were included in this prospective study, which was aimed to find out the common metabolic abnormality occurring in patients of this geographical region. The included variables were considered to be significant factors for the formation of stones if $\mathrm{p}<0.05$.

Out of these 100 patients studied in total, 73 were males and 27 were females. Thus, it was observed that in this study of small population, the frequency of stone formation was very high in males $(73 \%)$ compared to females $(27 \%)$ (Table 1$)$. The frequency of stone formation was high in the age group 51-60. The overall age range for stone formation in this study was observed to be 31-60 years (Table 2), and the mean age was observed to be $45.71 \pm 14.20$.

Abdominal pain was the major symptom along with fever and back pain among the patients. High BMI is assumed to be a risk factor for stone formation as $48 \%$ were belonging to the category of overweight and $7 \%$ were obese (Fig. 1).

About 55\% patients presented with bilateral stones and $12 \%$ with bilateral-recurrent stones (Table 3). Stone recurrence was present in around $28 \%$ patients showing that the FTSF was prone to the risk of recurrence. Thus, it is a good opinion to perform the metabolic evaluation in FTSF as well to reduce the recurrence of stones.

The majority of patients were non-vegetarians, i.e., $94 \%$ of the study population $(n=100)$ as shown in Table 4 . This shows that dietary pattern has a major role in stone formation. The individuals who were found to consume a high quantity of protein foods were at high risk. Thus, diet modification is essential. Average water intake was $<2$ liters/ day for majority, i.e., 73 patients (Table 5), which concluded that low water intake is another major risk factor for stone.

Several blood tests were performed to help detect the metabolic abnormality if any. Although urine analysis was not done for the entire population, it was performed in few individuals who had staghorn and

Table 1: Distribution of gender in the study population $(n=100)$

\begin{tabular}{ll}
\hline Gender & Frequency (\%) \\
\hline Male & $73(73.0)$ \\
Female & $27(27.0)$ \\
Total & $100(100.0)$ \\
\hline
\end{tabular}

Table 2: Distribution of different age groups in the study population $(n=100)$

\begin{tabular}{ll}
\hline Age & Frequency (\%) \\
\hline $10-20$ & $4(4.0)$ \\
$21-30$ & $7(7.0)$ \\
$31-40$ & $25(25.0)$ \\
$41-50$ & $20(20.0)$ \\
$51-60$ & $31(31.0)$ \\
$61-70$ & $9(9.0)$ \\
$71-80$ & $2(2.0)$ \\
$81-90$ & $2(2.0)$ \\
Total & $100(100.0)$ \\
\hline
\end{tabular}

Table 3: Distribution of stone type in the study population $(n=100)$

\begin{tabular}{ll}
\hline Type of stone former & Frequency (\%) \\
\hline Bilateral & $55(55.0)$ \\
Recurrent & $11(11.0)$ \\
Multiple & $7(7.0)$ \\
B/L and recurrent & $12(12.0)$ \\
B/L and multiple & $10(10.0)$ \\
Recurrent and multiple & $3(3.0)$ \\
B/L, recurrent and multiple & $2(2.0)$ \\
Total & $100(100.0)$ \\
\hline
\end{tabular}

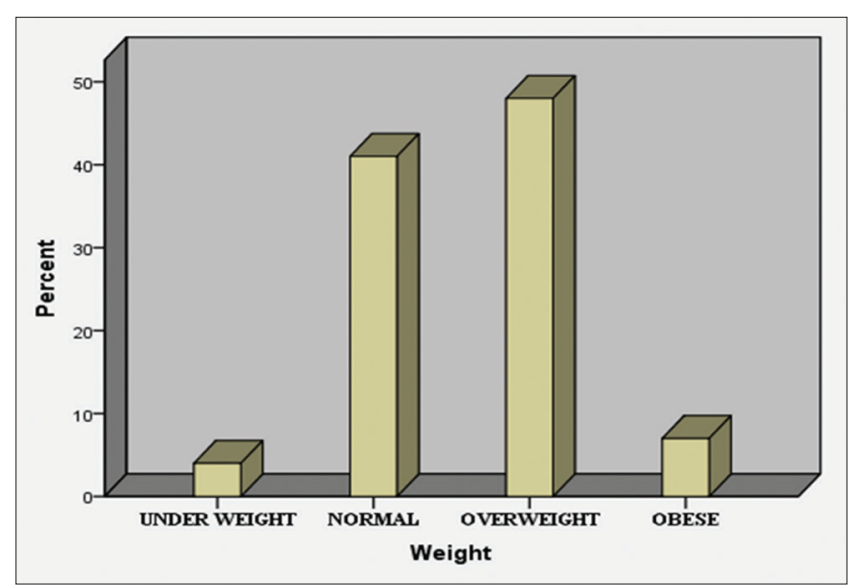

Fig. 1: A plot of weight in the study population $(n=100)$ 
multiple calculi. The main urine analysis components tested were urine volume, urine $\mathrm{pH}$, urine calcium, and urine uric acid. Patients who had acidic urine had a greater frequency of stone formation concluding that stone formation is greatly favored in acidic condition (Fig. 2). Thus, urine $\mathrm{pH}$ is also a risk factor and also it was statistically proven to be a significant factor $(\mathrm{p}=0.05$ in $\mathrm{n}=100)$ as shown in Table 6 . The urine calcium test was not performed in majority (94\%), and in the evaluated 6 patients, 2 were normal, 1 was low, and 3 were high (Fig. 3). In urine uric acid test, only $2 \%$ had increased levels (Fig. 4).

The individuals who showed an increased level of serum uric acid and calcium were not subject to urine calcium and uric acid test as the metabolic abnormality is already known.

Test for serum calcium was not done for $58 \%$ as there did not arise any need, it was normal for $36 \%$, low in $4 \%$, and high in $2 \%$ patients (Fig. 5), and statistically, it was proven to be a significant factor with stone formation in this small population under study $(\mathrm{p}=0.000$ in $\mathrm{n}=100$ and $\mathrm{p}=0.002$ in $\mathrm{n}=55$ ) as shown in Tables 6 and 7. Test for serum uric acid

Table 4: Distribution of diet in the study population $(n=100)$

\begin{tabular}{ll}
\hline Diet & Frequency (\%) \\
\hline Veg & $6(6.0)$ \\
Non-veg & $94(94.0)$ \\
Total & $100(100.0)$ \\
\hline
\end{tabular}

Table 5: Distribution of average water intake in the study population $(n=100)$

\begin{tabular}{ll}
\hline Average water intake & Frequency (\%) \\
\hline$<2 \mathrm{~L}$ & $73(73.0)$ \\
$2-3 \mathrm{~L}$ & $27(27.0)$ \\
Total & $100(100.0)$ \\
\hline
\end{tabular}

Table 6: Chi-square test for total population $(n=100)$

\begin{tabular}{ll}
\hline Variables & p value \\
\hline Sex & 0.734 \\
Weight & 0.437 \\
Family history & 0.016 \\
Alcohol intake & 0.101 \\
Diet & 0.670 \\
Average water intake & 0.984 \\
Urine volume & 0.124 \\
Urine pH & 0.050 \\
Urine calcium & 0.103 \\
Urine uric acid & 0.182 \\
Serum calcium & $<0.001$ \\
Serum uric acid & $<0.001$ \\
RBS & 0.028 \\
Serum creatinine & 0.040 \\
\hline
\end{tabular}

$\mathrm{p}<0.05$ significant. RBS: Random blood sugar

Table 7: Chi-square test for evaluated patients $(n=55)$

\begin{tabular}{ll}
\hline Variables & p value \\
\hline Sex & 0.435 \\
Diet & 0.759 \\
Weight & 0.346 \\
Urine volume & 0.032 \\
Urine pH & 0.152 \\
Calcium & 0.002 \\
Uric acid & $<0.001$ \\
Creatinine & 0.156 \\
RBS & 0.199 \\
\hline
\end{tabular}

$\mathrm{p}<0.05$ significant. RBS: Random blood sugar was not performed in 50 patients, it was normal in 36 patients, and high in 14 patients (Fig. 6) which showed that abnormal (increased) uric acid levels are statistically significant risk factor for stone formation $(\mathrm{p}<0.001$ in $\mathrm{n}=100$ and $\mathrm{p}<0.001$ in $\mathrm{n}=55)$ as in Tables 6 and 7 .

The patients RBS test when performed showed that $27 \%$ had high levels and 3 had greatly increased levels of blood glucose (Fig. 7). RBS was also observed to be a statistically significant factor for renal calculi

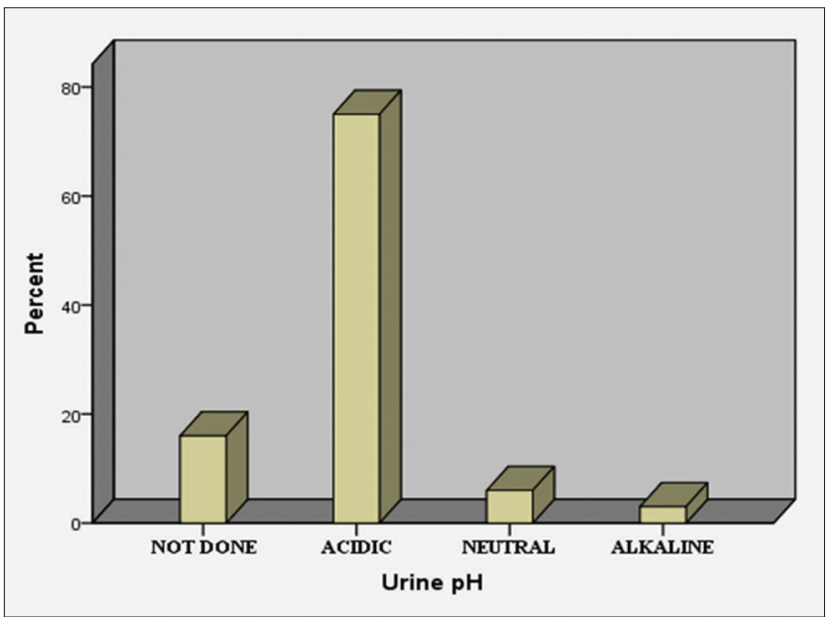

Fig. 2: Plot of urine $\mathrm{pH}(\mathrm{n}=100)$

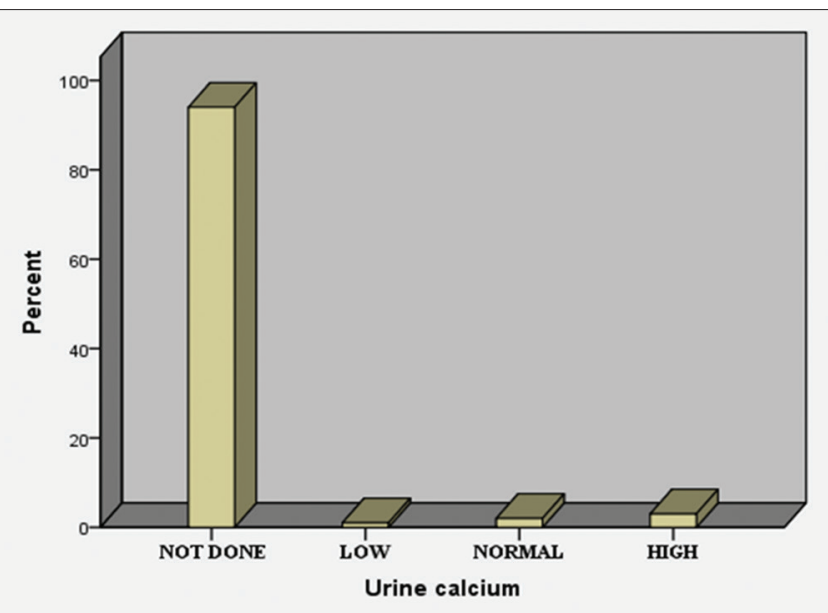

Fig. 3: Plot of urine calcium $(n=100)$

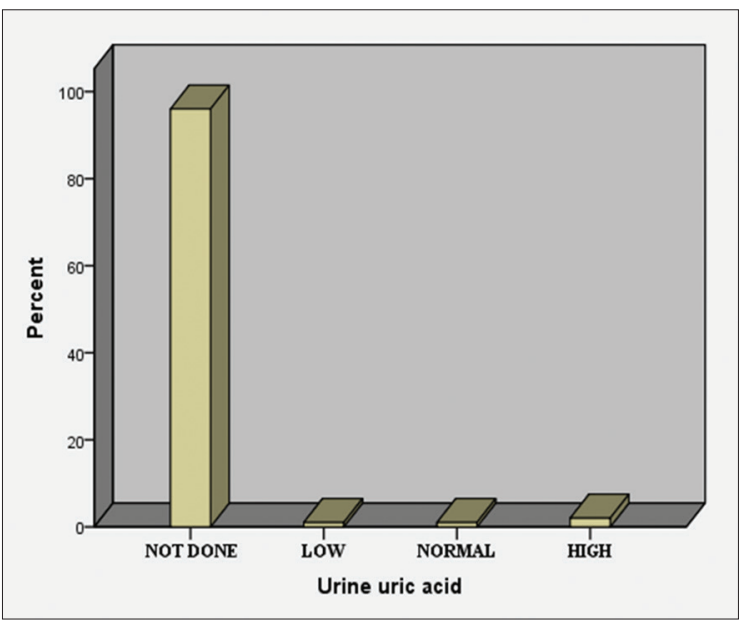

Fig. 4: Plot of urine uric acid $(n=100)$ 
( $\mathrm{p}=0.028$ in $\mathrm{n}=100)$ as in Table 6 . Serum creatinine tests revealed that the creatinine level was high in $26 \%$ and greatly increased in 12 patients, whereas it was normal in 61\% (Fig. 8). Creatinine was also a statistically significant factor $(\mathrm{p}=0.040$ in $\mathrm{n}=100)$ related to renal stones as shown in Table 6. BUN was abnormal in 12 patients (high in 11 and low in 1), normal in 21, and the test not performed in 67 patients (Fig. 9). Finally, it was observed that BUN also has significance with the stone formation ( $\mathrm{p}=0.03$ in $\mathrm{n}=100$ and $\mathrm{p}=0.052$ in $\mathrm{n}=55$ ) shown in Tables 6 and 7.

The major diagnostic tests done were computed tomography (CT) scan and ultrasound (US) scan and also a combination of US and CT scan was done for better and accurate stone confirmation results. The major treatment option in our hospital was a combination of ureterorenoscopy (URS)+extracorporeal shock wave lithotripsy (ESWL). URS alone was also a commonly used treatment option which gave satisfactory results and improved the quality of life of the patient.

Metabolic evaluation was done for 55 patients in the study population, and 23 out of this 55 evaluated were found to have a metabolic abnormality, whereas 32 were free from any abnormalities (Table 8). Out of this 55 patients, $40(72.7 \%)$ were males and $15(27.3 \%)$ were females (Table 9). 23 patients (41.8\%) had an abnormality in metabolism $(n=55)$. Metabolic abnormality was found in the age group 31-60 (Fig. 10). However, age was not a factor for metabolic abnormality. Gender did not also have significant difference ( $p=0.734$ in $n=100$ and $p=0.435$ in $n=55)$. Uric acid levels were high in 16 patients (29.1\%) as shown in Fig. 11, calcium levels high in 4 patients $(7.3 \%)$ as in Table 10 , and creatinine was high in 21 patients $(38.2 \%)$ as shown

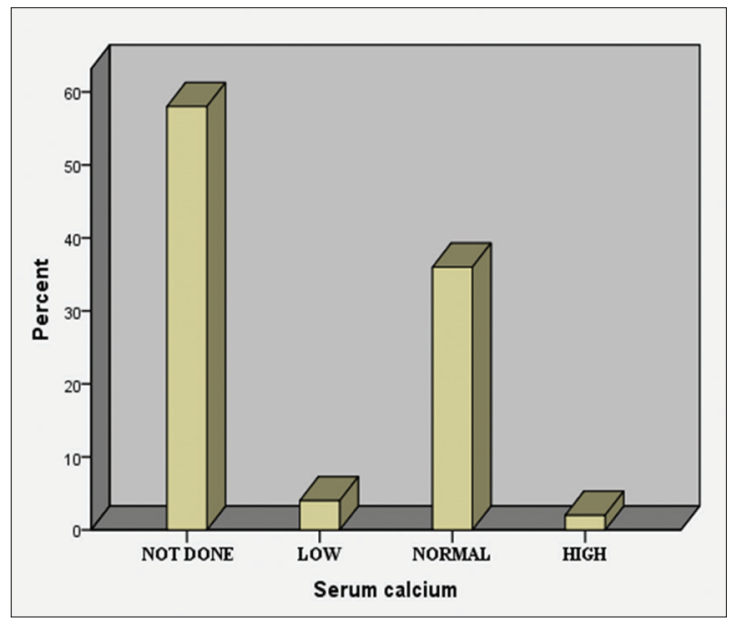

Fig. 5: Plot of serum calcium $(n=100)$

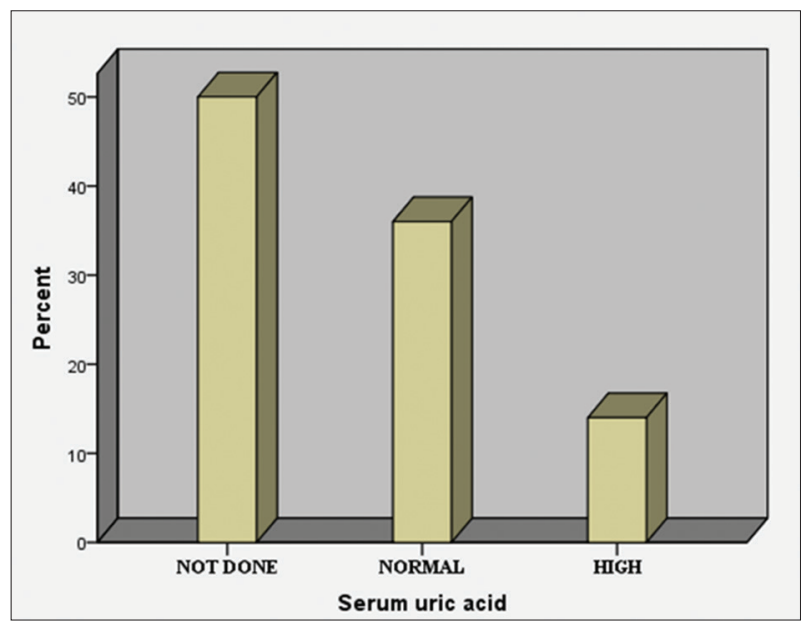

Fig. 6: Plot of serum uric acid $(n=100)$ in Table 11. In the evaluated population, urine volume was found to be low ( $\leq 1500 \mathrm{ml}$ ) in $25.5 \%$ and very low, i.e., $<1000 \mathrm{ml}$, in $5.5 \%$ of the patients (Fig. 12). Urine pH test was not done in $16.4 \%$ of the evaluated patients; it was acidic in $69.1 \%$, neutral in $10.9 \%$, and alkaline in $3.6 \%$ of the patients (Fig. 13).

Chi-square test was done to find out the significance of certain factors such as sex, diet, alcohol intake, weight, family history, urine volume, urine $\mathrm{pH}$, calcium, uric acid, RBS, and creatinine with stone formation. The test was individually applied to both the populations, i.e., $n=100$ as well as $n=55$. It was observed that sex, weight, diet, alcohol intake, and urine volume did not have a significant relationship with stone formation in the total population $(\mathrm{n}=100)$ studied. However, factors such as family history, urine $\mathrm{pH}$, serum calcium, serum uric acid, serum creatinine, and RBS were proved to be statistically significant factors of stone formation. Uric acid abnormality was found to be the major metabolic abnormality followed by calcium abnormality. Urine $\mathrm{pH}$ was also found to be a significant risk factor $(\mathrm{p}=0.050)$. Increased RBS and BUN also had contribution to stone formation (Table 6).

When Chi-square test was applied to the metabolically evaluated population, $n=55$, it was observed that increased uric acid was the major metabolic abnormality followed by increased calcium and decreased urine volume (Table 7). Therefore, it was found out from the study that hyperuricosuria, hypercalciuria, decreased urine volume, and acidic urine $\mathrm{pH}$ were the significant metabolic risk factors in the population evaluated.

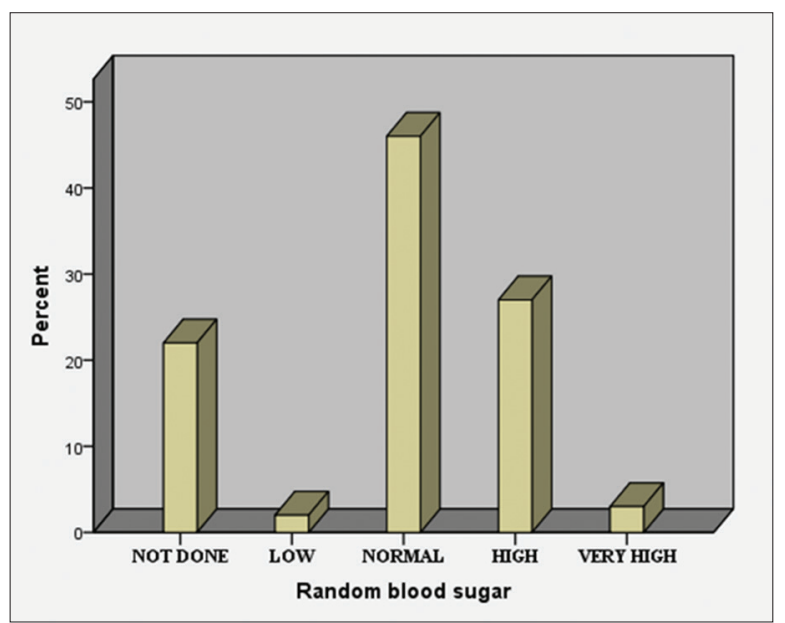

Fig. 7: Plot of RBS ( $n=100)$

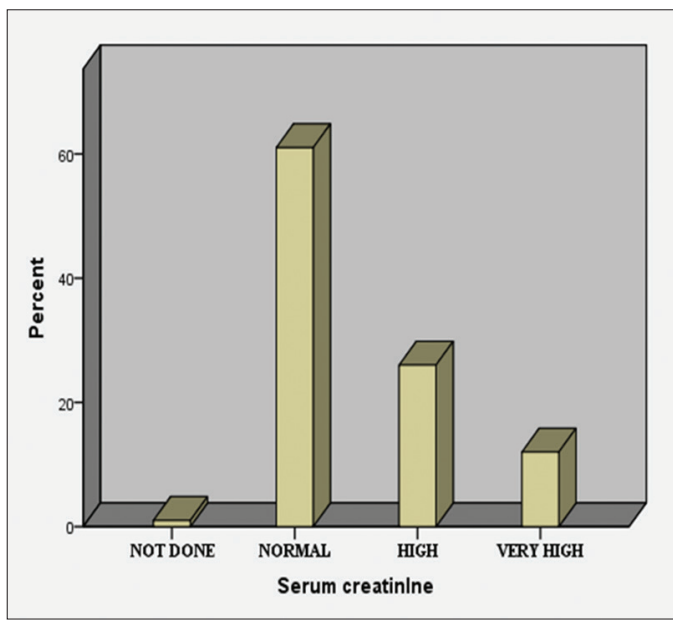

Fig. 8: Plot of serum creatinine $(n=100)$ 
Table 8: Metabolic evaluation done in the study population $(n=100)$

\begin{tabular}{ll}
\hline Metabolic evaluation & Frequency (\%) \\
\hline Not evaluated & $45(45.0)$ \\
Metabolic abnormality present & $23(23.0)$ \\
No metabolic abnormality & $32(32.0)$ \\
Total & $100(100.0)$ \\
\hline
\end{tabular}

Table 9: Distribution of gender in metabolically evaluated population $(n=55)$

\begin{tabular}{ll}
\hline Gender & Frequency (\%) \\
\hline Male & $40(72.7)$ \\
Female & $15(27.3)$ \\
Total & $55(100)$ \\
\hline
\end{tabular}

Table 10: Distribution of calcium level in the evaluated patients $(n=55)$

\begin{tabular}{ll}
\hline Calcium & Frequency (\%) \\
\hline not done & $11(20.0)$ \\
Low & $5(9.1)$ \\
Normal & $35(63.6)$ \\
High & $4(7.3)$ \\
Total & $55(100.0)$ \\
\hline
\end{tabular}

Table 11: Distribution of creatinine in the evaluated patients $(n=55)$

\begin{tabular}{ll}
\hline Creatinine & Frequency (\%) \\
\hline Not done & $1(1.8)$ \\
Normal & $33(60.0)$ \\
High & $21(38.2)$ \\
Total & $55(100.0)$ \\
\hline
\end{tabular}

\section{DISCUSSION}

Nephrolithiasis or stone disease has become a relatively common disorder, and its prevalence is greatly increasing worldwide. Here, in this geographical area, renal stone patients are common, and we have plenty of them consulting the urology department of our hospital. In this study, the major focus was given to patients who were diagnosed with bilateral, recurrent, and multiple stones, and single stone formers were excluded as this study was conducted to evaluate the metabolic abnormalities related to stone formation. Single stones occur as a result of either structural abnormality of the urinary tract or lifestyle pattern of the patients such as low water intake, high protein intake, occupation in hot places, and obesity. Single stone usually is not related to any metabolic abnormality.

Any metabolic abnormality in an individual can result in staghorn calculi or multiple/bilateral stone. If this abnormality is left untreated, it can lead to recurrence of stones. The common metabolic abnormalities occurring in such patients are hypercalciuria, hyperuricosuria, hyperoxaluria, and hypocitraturia. An individual can have one or more than one metabolic abnormality. Thus, evaluation of the metabolic abnormality and treating the same can greatly reduce the incidence of stones.

In case of single stone or FTSF, if the stone is staghorn calculi, it is always good to perform a metabolic evaluation, so as to help the individual cope up with the risk of further recurrences in the future. This fact is supported by the study conducted by Joshi et al. [7], in which he finally concluded that comprehensive metabolic evaluation is required even

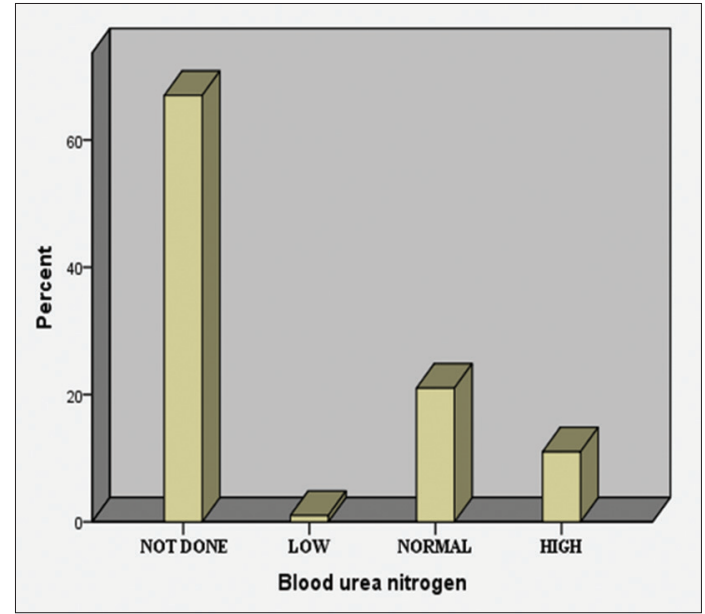

Fig. 9: Plot of BUN $(n=100)$

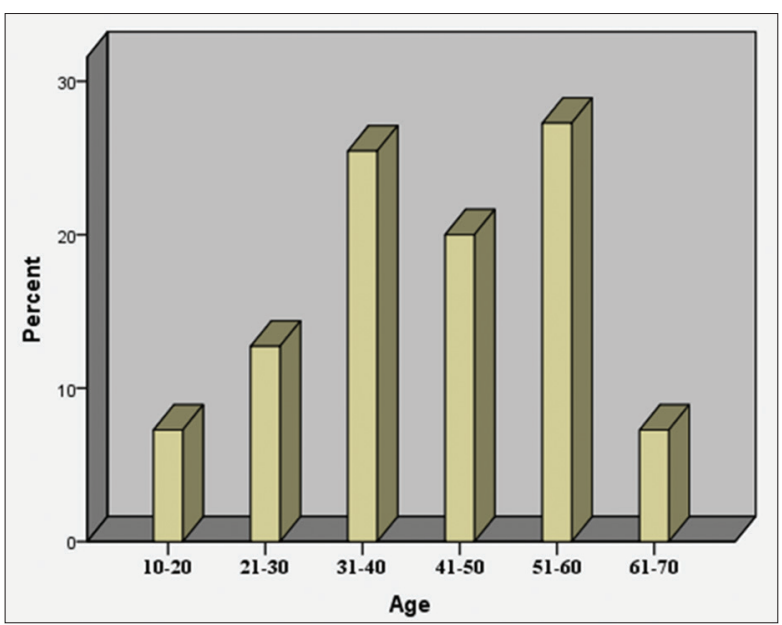

Fig. 10: Plot of age in $n=55$

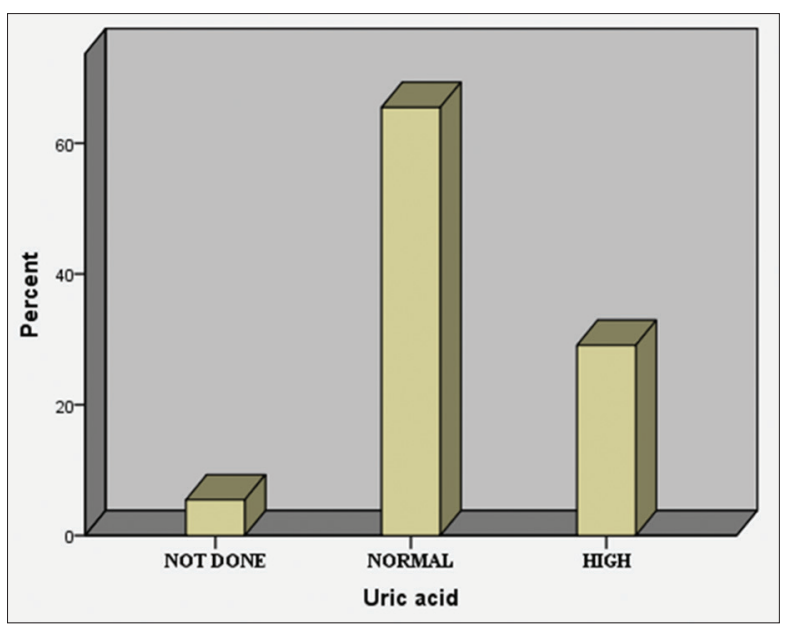

Fig. 11: Plot of uric acid in $n=55$

in FTSF. By observing the etiology of stone formation, we can greatly decrease the possibility of recurrent stone formation with medical therapy. Thus, it is possible to reduce the morbidity, mortality, and cost associated with renal stones.

All stone forming patients should have a thorough history and physical examination, where any previous history of stone disease and treatment 


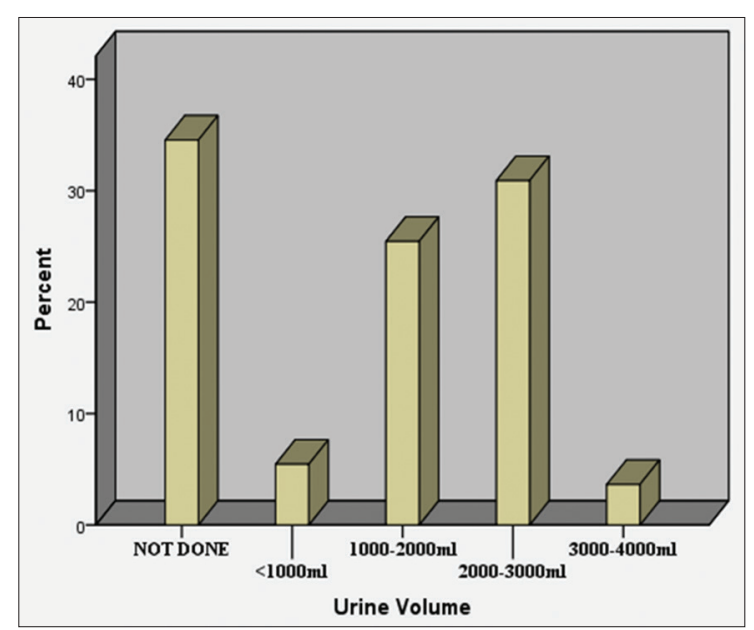

Fig. 12: Plot of urine volume in $n=55$

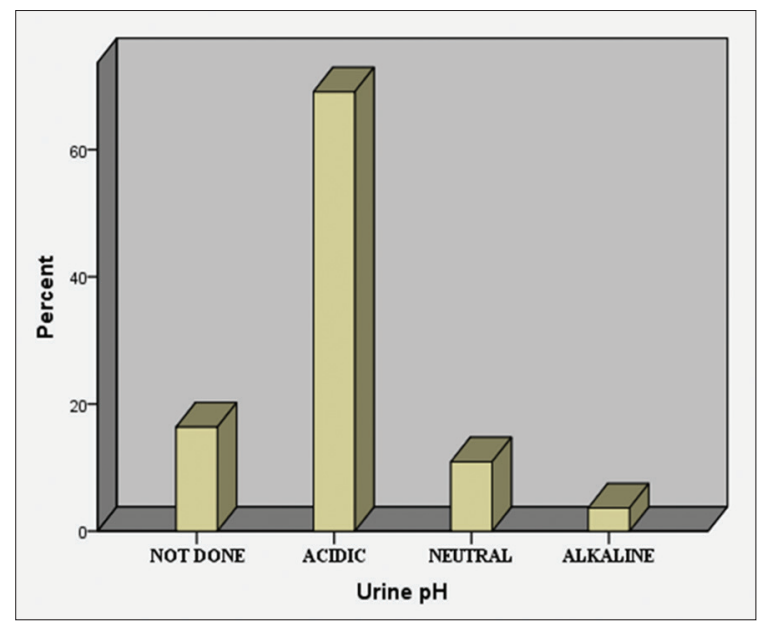

Fig. 13: Plot of urine $\mathrm{pH}$ in $\mathrm{n}=55$

along with family history, if any, should be documented. In this study, it was observed that family history did have a significant relationship with stone formation. Thus, it is concluded that there are chances of calculi formation in first or second generations of the stone former.

In this study, $48 \%$ patients were overweight, whereas $7 \%$ were obese, which shows that increased body mass can be another causative factor for renal stones. Although weight was not proved to be statistically significant, it could be a contributing factor toward stone formation. Moreover, this was in accordance with the large prospective study conducted by Taylor et al. [8], in which it was concluded that obesity/ weight gain can increase the risk of renal stone formation and that the magnitude of increased risk may be greater in women than in men (in the present study, the magnitude of increased risk was greater in men). Weight gain since early adulthood was associated with an increased risk of incident kidney stone formation in both men and women. Another study conducted by Charkhchian et al. [9] also revealed that higher BMI may be a risk factor for nephrolithiasis.

Shekarriz and Marshall [10] in his study suggested that laboratory evaluation should include urine analysis and culture; stone analysis is an integral part of evaluation. Some authors recommend metabolic evaluation in all patients even those with a single stone episode, whereas others suggest that single stone formers without any risk factors do not require further investigations. In patients with high risk for stone recurrence or recurrent stone former, a more detailed evaluation is recommended where additional $24 \mathrm{hrs}$ urine collection with calcium and uric acid tests should be done.
Treatment of renal stones is of much importance, and medical therapy helps reduce recurrence of stones significantly. Medical expulsive therapy is used for spontaneous stone passage, and it has been provided in our hospital. In cases where medical expulsive therapy fails to pass the stone, other treatment opinions such as URS, ESWL, percutaneous nephrolithotomy (PCNL), and surgery are taken into consideration. Usually for patients presenting hyperuricosuria, allopurinol, which is a xanthine oxidase inhibitor, is the drug of choice and for those presenting hypercalciuria, treatment with thiazides are preferred. Appropriate antibiotic therapy is also prescribed in cases which show stones with infection.

The present study concluded that hyperuricosuria, low urine volume, and acidic urine $\mathrm{pH}$ were significant risk factors of stone formation, and the results of this study perfectly complies with the former study conducted by Xu et al. [11], in which he explained that low urine volume, hyperuricosuria, and abnormally acidic urine $\mathrm{pH}$ leads to formation of uric acid stone. Hyperuricosuria can occur as a result of intake of purine-rich diet, low water intake, and acidic urine $\mathrm{pH}$. Low urine $\mathrm{pH}$ is the principal determinant in uric acid crystallization. In such condition, the main aim of treatment is to increase the solubility of uric acid in urine and to reduce its concentration by urinary alkalization using sodium or potassium alkali. The consumption of animal protein should also be greatly reduced.

Renal stones are usually preventable. In a study conducted by Goldfarb and Arowojolu [12], it was said that, to maximize the efficacy of preventive regimens and to obtain a cost-effective treatment, the appropriate data should be collected (past medical history, family history, social history, occupation, activities, and diet). Laboratory evaluation of serum chemistries, urine analysis, and 24 hrs urine collections are most appropriate for recurrent stone formers. Past history and family history details were collected and observed in the present study; family history was found to be significant, whereas history did not show to be significant. Hypertension and diabetes are often associated with metabolic syndrome, which in turn is associated with an increased risk of uric acid stone formation caused by the associated unduly acid urine caused by insulin resistance. In the present study, it was observed that diabetes and hypertension were predominant past medical history, and thus, they maybe a contributor to stone formation. Talking about diet, although majority of the study population was non-vegetarians (94\%), a non-vegetarian dietary pattern did not prove to be a statistically significant risk factor as per Chi-square test. However taking in consideration the total population, it is suggested that high intake of animal protein can lead to significantly increased the risk for stone formation.

It was also found out in this study that complete metabolic evaluation was not performed in the majority of the patients, which was similar to a study conducted by Krepinsky et al. [13], in which he concluded that the majority of patients presenting with recurrent stones do not actually undergo appropriate metabolic investigations. The metabolically evaluated patients $(n=55)$ of this present study were also not completely evaluated as 24 hrs urine analysis was not compulsorily performed by the physicians. Only certain variables such as serum calcium, serum uric acid, urine $\mathrm{pH}, \mathrm{RBS}$, creatinine, and BUN were performed, and patients who met these tests were considered metabolically evaluated. In certain cases, where blood tests did not reveal any major abnormality, 24 hrs urine collections were made and test for urine volume, urine $\mathrm{pH}$, urine calcium, urine uric acid, and urine creatinine was investigated. These patients were known to be completely evaluated, and such evaluation will surely prove to be worth regarding a stone former since the underlying problem can be identified and easily tackled. On the other hand, where complete metabolic evaluation is not ordered, it may not be possible to explain the major abnormalities present in the patients and the cause for such an abnormality. Thus, it is strongly suggested that the patients presenting with staghorn, multiple, or recurrent calculi must be subject to complete metabolic evaluation and strategies must be undertaken to implement the same in all major health-care centers. 


\section{CONCLUSION}

Out of the 100 patients included in the study, only 55 patients were subject to metabolic evaluation. The rest 45 patients were given proper treatment, mainly URS, ESWL, PCNL, and a combination of URS and ESWL/PCNL, and they left the hospital in a stable condition. The reason for not performing the metabolic evaluation in the $45 \%$ patients can be multifactorial. It might be because of poor patient compliance due to low economic status, to avoid unnecessary evaluation cost, or undervaluation as the patients did not come for further follow-up.

Out of the 55 patients evaluated, the metabolic abnormality was observed in 23 patients $(41.8 \%)$. Once there is any metabolic abnormality, there is chance for the individual to get stones in both the kidneys. The FTSF who may be diagnosed with bilateral or multiple stones should also be metabolically evaluated, to prevent further recurrence.

As per the study, hyperuricemia/uricosuria (29.1\%) was found to be the major metabolic abnormality in the evaluated patients $(n=55)$ followed by hypercalcemia/calciuria (7.3\%). A low urine volume and acidic urine $\mathrm{pH}$ were also considered to be contributing risk factors. Even though non-vegetarian diet and low water intake were major risk factors for renal stones, the statistical method used did not show any significance. This might be due to the small sample size used in the study which was not sufficient for an accurate result. This only shows the pattern of stone formation in the patients studied and not for the entire renal stone population. Abnormality in uric acid level was statistically significant $(p=0.000)$. Abnormal calcium $(p=0.002)$ and creatinine $(p=0.040)$ levels were also statistically significant. Even though there is no direct correlation between increased RBS and renal stones, in this study, it showed statistical significance, and thus, it might be associated with hyperuricemia in stone patients. There might be an indirect relationship between hyperglycemia and hyperuricemia in individuals, and hypothetically, most of the diabetic patients had hyperuricemia that explains stone incidence. Thus, any abnormality in RBS levels of an individual should also be treated to avoid stones as well as other critical medical conditions such as diabetes and metabolic syndrome.

For the rest of the patients (58.2\%), who did not have any metabolic abnormality, the major risk factors were assumed to be non-vegetarian diet and low water intake. The hot climate of this geographical region is also a possible contributor to stone formation and recurrence. Thus, a proper diet recommendation is to be provided, and the patient should be explained about the importance of drinking adequate water, i.e. a stone former must drink more than 3 liters of water a day and with physical activity and hot climate, the intake should be increased.

It was reported that hyperuricemia/uricosuria was the major metabolic abnormality in this geographical region, followed by acidic urine $\mathrm{pH}$, low urine volume, and a small percentage of people showed hypercalcemia/calciuria. It was also reported that renal stone disease was not related to any age group, though in this population studied, it was more common in the age group of 51-60 and the mean age of stone formation was observed to be $45.71 \pm 14.20$. It occurs in both gender, but the incidence was high in males than in females. Thus, in this study, renal stone formation showed no association with the patient's age and gender. The metabolic abnormalities were corrected by administration of suitable medications and proper patient care. Proper treatment was provided in the hospital to ensure that the patients left in a stable and healthy condition, along with patient education so as to reduce the recurrence of stones as well as economic costs. Patients should be taught about the role of diet and water intake in the prevention of metabolic abnormalities and thereby prevention of renal stones. The patients were suggested to go with very high fluid intake along with reduction of amount of protein and salt in the diet. Finally, it is suggested that those presenting with renal stones should be robustly evaluated so as to improve the quality of treatment as well as the quality of life of the patients.

In conclusion, it was finalized that any patient with multiple or recurrent stones will surely have one or more metabolic abnormality that can be treated by providing good medical care. It was also observed that stone patients were under evaluated in the hospital, which is either due to the patients low financial background or because these patients did not return for further follow-up. Implementation of metabolic evaluation in patients presenting with staghorn, multiple, or recurrent stones is compulsory, and it will definitely help in preventing further recurrences. Long-term studies to evaluate stone patients for metabolic abnormalities should be taken into account so as to significantly reduce the prevalence and incidence of stones in the general population. Improved awareness about modifiable risk factors of renal stones among both general population and the health-care providers is very necessary, and this can be achieved by providing proper education regarding the same. This helps in improving the general health and decrease morbidity and mortality and even the cost associated with the renal stone disease.

\section{ACKNOWLEDGMENT}

I would like to express my deep sense of gratitude and thanks to all the physicians and staffs of the Department of Urology, Kovai Medical Center and Hospital, Coimbatore. I also express my appreciation to all the teaching staffs of the Department of Pharmacy Practice, KMCH College of Pharmacy, for their incredible guidance.

\section{REFERENCES}

1. Kummer AE, Grams M, Lutsey P, Chen Y, Matsushita K, Köttgen A et al. Nephrolithiasis as a risk factor for CKD: The atherosclerosis risk in communities study. Clin J Am Soc Nephrol 2015;10(11):2023-9.

2. Guy's and St Thomas' NHS Foundation Trust, 2014; Leaflet No. 2390/VER3.

3. Thorsten B, Murat B, Nicola D, Bente TJ, Thomas K, Andre M, et al. Patient information - Metabolic evaluation for kidney and ureteral stones. EAU 2012:1-2.

4. Al-Risi AO, Nida'a MA, Alka A. Study on prevalence and management of renal stones among Omani in-patients at Sohar hospital. SJAMS 2014:2(1A):22-33.

5. Andreas S, Michael S, Thomas K, Kemal S, Christian S, Ales P, et al. Metabolic Evaluation and recurrence prevention for urinary stone patients: EAU guidelines. Eur Urol 2015;67:750-63.

6. Paterson RF. Arguments for a comprehensive metabolic evaluation of the first-time stone former. Can Urol Assoc J 2010;4(3):209-10.

7. Joshi A, Gupta SK, Srivastava A. Metabolic evaluation in first-time renal stone formers in North India: A single center study. Saudi J Kidney Dis Transpl 2013;24(4):838-43.

8. Taylor EN, Stampfer MJ, Curhan GC. Obesity, weight gain, and the risk of kidney stones. JAMA 2005 26;293(4):455-62.

9. Charkhchian M, Samani S, Merat E. Clinical and metabolic evaluation of patients with history of renal calculi in Qazvin, Iran. Ir J Med Sci 2015;184(4):731-5.

10. Shekarriz B, Marshall LS. Metabolic evaluation of stone disease. Braz J Urol 2001;27(1):10-8.

11. Xu H, Zisman AL, Coe FL, Worcester EM. Kidney stones: An update on current pharmacological management and future directions. Expert Opin Pharmacother 2013;14(4):435-47.

12. Goldfarb DS, Arowojolu O. Metabolic evaluation of first-time and recurrent stone formers. Urol Clin North Am 2013;40(1):13-20.

13. Krepinsky J, Ingram AJ, Churchill DN. Metabolic investigation of recurrent nephrolithiasis: Compliance with recommendations. Urology 2000;56(6):915-20. 